\title{
Medição da versão acetabular após artroplastia total do quadril: Comparação de dois métodos radiográficos*
}

\section{Measuring Acetabular Version after Total Hip Arthroplasty: A Comparison of Two Radiographic Methods}

\author{
Afonso Cardoso ${ }^{10}$ Mafalda Duarte ${ }^{2}$ Rui Viegas ${ }^{10}$ Filipe Oliveira $^{1}$ Pedro Pinto $^{1}$ Paulo Rego ${ }^{1}$ (1) \\ ${ }^{1}$ Departamento de Ortopedia e Traumatologia, Hospital Beatriz \\ Ângelo, Loures, Portugal \\ 2 Faculdade de Medicina da Universidade de Lisboa, Lisboa, Portugal \\ Endereço para correspondência Afonso Nobre Pinto Cardoso, MD, \\ Departamento de Ortopedia e Traumatologia, Hospital Beatriz \\ Ângelo, Av. Carlos Teixeira, 514, 2674, Loures, Portugal \\ (e-mail: afonso.cardoso@hbeatrizangelo.pt).
}

Rev Bras Ortop 2021;56(4):513-516.

\section{Resumo \\ Palavras-chave \\ - artroplastia de quadril \\ - acetábulo \\ - radiografia}

Objetivo O objetivo do presente trabalho foi comparar a medição da versão do componente acetabular em radiografias em incidência anteroposterior (AP) e crosstable após artroplastia total do quadril (ATQ).

Métodos Foram selecionadas radiografias de 60 quadris com ATQ primário. A versão foi calculada na radiografia AP usando o método de Lewinnek e, na cross-table, usando o método direto do Woo e Morrey.

Resultados A média e o desvio padrão (DP) foram diferentes em ambas as radiografias, sendo $9,7^{\circ} \pm 5,5^{\circ}$ no AP, enquanto na cross-table foram $20,6^{\circ} \pm 8,4^{\circ}$ $(p<0,001)$. Considerando nosso objetivo de $10^{\circ}$, as medidas da cross-table foram estatisticamente diferentes dele $(p<0,001)$, enquanto a medição AP não diferiu $(p=0,716)$.

Conclusão O presente estudo mostrou que a melhor maneira de avaliar corretamente o posicionamento do componente acetabular após uma ATQ é medindo a anteversão e a abdução em uma radiografia AP após confirmar, em uma radiografia cross-table, que o componente não é retrovertido.

Trabalho desenvolvido no Departamento de Ortopedia e Traumatologia do Hospital Beatriz Ângelo, Loures, Portugal.

recebido

10 de Março de 2020

aceito

16 de Setembro de 2020

Publicado on-line

Março 22, 2021
DOI https://doi.org/

10.1055/s-0040-1721360. ISSN 0102-3616. (c) 2021. Sociedade Brasileira de Ortopedia e Traumatologia. All rights reserved.

This is an open access article published by Thieme under the terms of the Creative Commons Attribution-NonDerivative-NonCommercial-License, permitting copying and reproduction so long as the original work is given appropriate credit. Contents may not be used for commercial purposes, or adapted, remixed, transformed or built upon. (https://creativecommons.org/ licenses/by-nc-nd/4.0/)

Thieme Revinter Publicações Ltda., Rua do Matoso 170, Rio de Janeiro, RJ, CEP 20270-135, Brazil 


\author{
Keywords \\ - arthroplasty, \\ replacement, hip \\ - acetabulum \\ - radiography
}

Results Mean and standard deviation (SD) were different on both radiographs, being $9.7^{\circ} \pm 5.5^{\circ}$ on the AP, whereas in the cross-table the measurements were $20.6^{\circ} \pm 8.4^{\circ}$ $(p<0.001)$. Minding our aim of $10^{\circ}$, the cross-table measurements were statistically different from it $(p<0.001)$, while the AP measurement did not differ $(p=0.716)$. Conclusion The present study showed that the best way to correctly evaluate the acetabular component positioning following a THA is by measuring anteversion and abduction on an AP radiograph after confirming, in a cross-table radiograph, that the component is not retroverted.

\section{Introdução}

A estabilidade é um dos fatores e objetivos mais importantes em uma artroplastia total do quadril (ATQ) e, para alcançá-la, a colocação correta do componente é de suma importância. ${ }^{1}$ $\mathrm{O}$ posicionamento ruim do componente acetabular tem se mostrado uma causa importante de luxação, e também leva a uma limitação do movimento e ao aumento do desgaste do polietileno. ${ }^{2-4}$ A posição acetabular é definida por sua abdução e sua versão. ${ }^{5} \mathrm{~A}$ abdução é definida como o ângulo entre a face do copo e o eixo transversal, enquanto a versão é definida como o ângulo entre o eixo do componente e o plano coronal. ${ }^{5} \mathrm{~A}$ importância desses parâmetros reside no reconhecimento de que diferenças sutis de orientação podem levar a uma taxa de deslocamentos maior. ${ }^{6}$ Lewinnek et al. sugerem que um componente acetabular ideal deve ter um abdução de $40^{\circ}$ e uma anteversão de $15^{\circ} .7$ A abdução acetabular é relativamente fácil de medir em radiografias anteroposteriores (APs), mas isso não é verdade para a versão. Inúmeros estudos têm se concentrado em diferentes métodos para medir a versão em radiografias, embora nem todos tenham se mostrado consistentes e precisos. ${ }^{5,7-12} \mathrm{Um}$ método ideal deve ser preciso, reprodutível e viável dentro de uma prática cotidiana. Para medir a abdução, usa-se uma radiografia AP pélvica, enquanto que para medir a versão, os métodos consistem em diferentes equações matemáticas que usam radiografias AP ou uma medida direta em uma radiografia cross-table. ${ }^{5,7-12}$ Embora seja verdade que a medição da versão é simples com radiografias cross-table, essas radiografias muitas vezes dependem de imagens de boa qualidade que podem ser comprometidas pela rigidez da articulação do quadril contralateral. ${ }^{13}$

O objetivo do presente trabalho foi comparar a medida da versão do componente acetabular em uma radiografia AP usando o método Lewinnek e a medida direta em uma radiografia cross-table. Nossa hipótese é a de que a radiografia cross-table é mais influenciada pelo posicionamento do paciente, o que poderia levar a uma medida incorreta da versão acetabular.

\section{Material e Métodos}

Entre junho de 2018 e julho de 2018, os pacientes que compareceram ao hospital foram examinados para inclusão no presente estudo. Os critérios de inclusão foram ATQ primários para coxartrose primária feitos em nossa institui- ção. Foram excluídos pacientes com histórico de cirurgia instrumentada na coluna vertebral. Os pacientes selecionados foram submetidos a um AP pélvico e a uma radiografia cross-table. $\mathrm{O}$ estudo foi aprovado pelo comitê de ética da instituição e o consentimento informado foi obtido de todos os pacientes. Em todos os pacientes, foi utilizado o mesmo componente acetabular (Epifit, Smith \& Nephew, Londres, Reino Unido). Utilizamos o guia de alinhamento, de acordo com a técnica cirúrgica, para colocar o componente acetabular em $10^{\circ}$ de anteversão.

Todas as radiografias foram tiradas no mesmo departamento de radiologia de acordo com um protocolo padrão. As radiografias AP pélvicas foram feitas na posição supina, com ambos os quadris estendidos, com uma distância de imagem de origem de $100 \mathrm{~cm}$ do filme, perpendicular ao paciente, centrada no aspecto superior da sínfise púbica. As radiografias laterais cross-table foram tomadas com o quadril contralateral flexionado o máximo possível até $90^{\circ}$, com o raio central inclinado perpendicularmente ao eixo longo do pescoço do fêmur ( $45^{\circ}$ cefálico), paralelo à mesa de exame. Todas as imagens foram adquiridas, e todas as medições foram feitas utilizando o software Picture Archiving and Communication System (PACS) (syngo.plaza, Siemens Healthineers, Erlangen, Alemanha).

A versão foi calculada na radiografia AP usando o método de Lewinnek. ${ }^{7}$ Neste método, utiliza-se a elipse formada pelo componente acetabular para que a versão seja $=\operatorname{sen}^{-1}$ (eixo curto/eixo longo) (-Fig. 1). Na radiografia cross-table, a

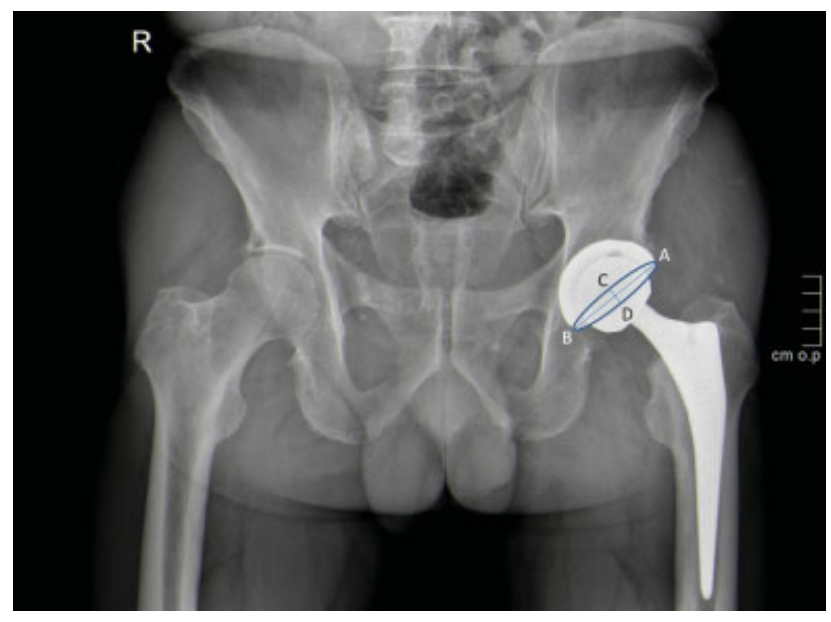

Fig. 1 Representação do método de Lewinnek: versão $=\operatorname{sen}^{-1}(A B / C D)$. 


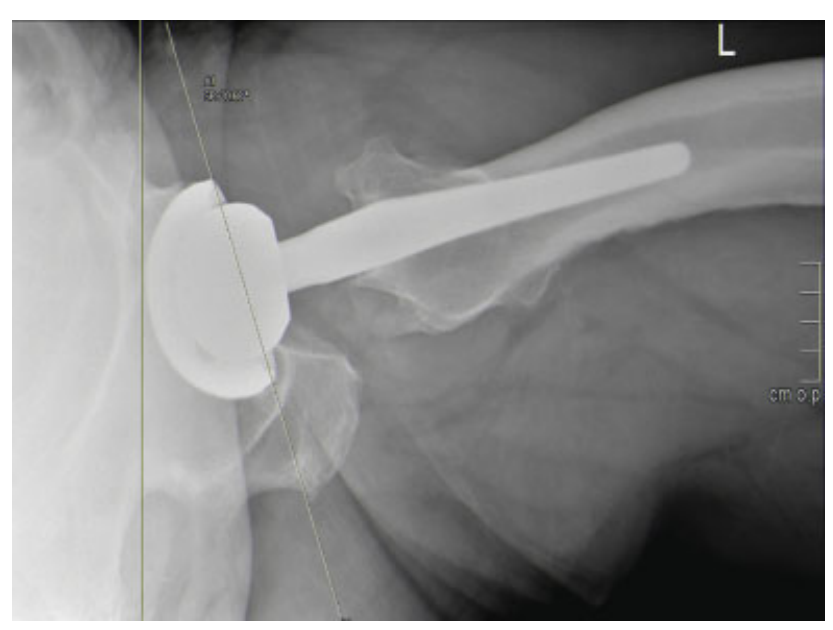

Fig. 2 Representação do método de Woo e Morrey: versão = ângulo entre uma linha perpendicular e uma linha tangencial à face de abertura do componente acetabular.

versão foi o ângulo medido entre uma linha perpendicular à mesa de exame e uma linha tangencial à face de abertura do componente acetabular, conforme descrito por Woo et al. ${ }^{4}$ (-Fig 2). A radiografia cross-table também foi analisada com o objetivo de excluir a retroversão, que seria indetectável nas radiografias $A P$.

A normalidade dos dados foi testada com o teste de Kolmogorov-Smirnov. Os dados foram comparados e analisados utilizando-se o teste- $t$ de Student para uma amostra e amostra dependente, e o teste de Mann-Whitney ou Wilcoxon, de acordo com a normalidade dos dados. A correlação dos dados foi analisada com o coeficiente de correlação de Pearson ou Spearman de acordo com sua normalidade. As variáveis nominais foram comparadas utilizando-se o teste qui-quadrado. Valores-p $<0,05$ foram considerados significativos. Um software estatístico dedicado (IBM SPSS Statistics for Windows, versão 21, IBM Corp., Armonk, NY, EUA) foi utilizado.

\section{Resultados}

Identificamos e incluímos 54 pacientes (60 quadris). Havia 36 homens e 18 mulheres e a média de idade dos pacientes em cirurgia era de 68,5 anos. Não foram encontradas diferenças entre as medidas e idade ou gênero. $O$ seguimento médio foi de 45,3 meses ( 24 a 71 meses).

Para a medida de anteversão pelo método Lewinnek em radiografias AP, a média e o desvio-padrão (DP) foram $9,7^{\circ} \pm 5,5^{\circ}\left(2^{\circ}-21^{\circ}\right)$. Para a medida de anteversão em radiografias cross-table, a média e o DP foram de $20,6^{\circ} \pm 8,4^{\circ}\left(0^{\circ}-\right.$ $40^{\circ}$ ). Como tal, não houve casos de retroversão. Houve correlação positiva entre ambos os métodos $(n=60, \mathrm{r}=0,642$, $p<0,0001)$. No entanto, ambas as medidas deram resultados estatisticamente diferentes $(p<0,001)$. A média e o DP para a diferença de ambos os métodos foram de $10,8^{\circ} \pm 6,6^{\circ}\left(-13^{\circ}-\right.$ $29^{\circ}$ ). Considerando nosso objetivo de $10^{\circ}$ de anteversão, testamos ambas as medidas contra esse valor e descobrimos que a medição cross-table era estatisticamente diferente dele $(p<0,0001)$, enquanto a medição AP não difere $(p=0,716)$.

\section{Discussão}

Após a ATQ existem vários exames de imagem que podem ser realizados para avaliar o estado da artroplastia e a posição dos componentes. Embora exames como tomografia computadorizada (TC) possam ser mais precisos, eles representam aumento de custos e radiação para o paciente. Portanto, a radiografia tradicional continua sendo o exame mais importante no acompanhamento das artroplastias.

O posicionamento dos componentes é essencial para sua estabilidade, função e longevidade. ${ }^{2,3,7}$ No entanto, como alcançá-lo, ou seja, quanto dar de anteversão acetabular, permanece controverso. Vários autores recomendam entre $0^{\circ}$ e $30^{\circ}$ de anteversão. ${ }^{1,14,15}$ Em nosso centro, buscamos uma anteversão de $10^{\circ}$. Embora o conceito de zona segura de Lewinnek tenha guiado o posicionamento de componentes acetabulares por muitos anos, há evidências de que uma importante proporção de ATQ se desloca dentro dessa zona segura. ${ }^{16,17}$ Além disso, a anteversão acetabular não deve ser analisada por conta própria, pois a combinação das anteversões femoral e acetabular pode prever melhor o risco de luxação. ${ }^{18}$ Por outro lado, outros autores tentam colocar o componente acetabular em posição anatômica de acordo com referências anatômicas específicas do paciente (como o ligamento acetabular transversal). ${ }^{19,20}$ Qual dos dois métodos produz melhores resultados, no entanto, ainda é uma questão de debate. Os cirurgiões devem ter em mente que a estabilidade de uma ATQ é uma interação entre a anatomia específica do paciente e o posicionamento de componentes, e também depende da restauração adequada da tensão e do equilíbrio do tecido mole.

Considerando que é fácil medir a abdução acetabular, não se pode dizer o mesmo para a anteversão. Nho et al. estudaram vários métodos e concluíram que os métodos de Lewinnek, Hassan e Liaw, baseados em radiografias AP, eram semelhantes às medições feitas em TCs. ${ }^{10} \mathrm{Em}$ seu estudo, eles também incluíram o método de Woo e Morrey para radiografias cross-table que também deram resultados semelhantes. ${ }^{10}$ No entanto, outro estudo de Arai et al. encontrou uma diferença de $5^{\circ}$ entre as medidas de AP e de crosstable. ${ }^{13}$ Além disso, e talvez mais clinicamente relevante, eles também encontraram uma correlação entre um quadril duro contralateral e a medição da versão acetabular na radiografia de cross-table devido à inclinação pélvica. ${ }^{13}$

Em nosso estudo, encontramos uma anteversão acetabular média de $9,7^{\circ} \pm 5,5^{\circ}$ ao usar o método de Lewinnek em uma radiografia AP, o que não é significativamente diferente da nossa meta de $10^{\circ}$. Por outro lado, utilizando a radiografia cross-table, medimos uma anteversão de $20,6^{\circ} \pm 8,4^{\circ}$, o que difere significativamente do nosso alvo cirúrgico. Ambas as medidas foram estatisticamente diferentes umas das outras. Observamos também uma gama mais ampla de medições nas radiografias transversais $\left(40^{\circ}\right.$ versus $\left.19^{\circ}\right)$. Encontramos uma correlação moderada entre ambos os métodos que está abaixo das expectativas ao medir o mesmo parâmetro.

A radiografia cross-table, quando o posicionamento adequado é enfatizado, tem sido mostrada para determinar com precisão a anteversão acetabular. ${ }^{21}$ No entanto, dados os resultados do presente estudo, acreditamos que este método 
é propenso à imprecisão, devido ao mau posicionamento, provavelmente relacionado ao quadril contralateral ou rigidez lombar. Além disso, é nossa opinião que a inclinação pélvica é mais difícil de detectar em radiografias cross-table do que em incidências de AP, o que impede erros negligentes nessa última, mas não na primeira. Não obstante, as radiografias AP têm algumas limitações porque a retroversão não pode ser detectada e o ápice da elipse é um pouco difícil de identificar quando um forro de metal é usado.

Quanto às limitações do nosso estudo, um padrão de medição, como uma TC, poderia fortalecer ainda mais nossas conclusões.

\section{Conclusão}

O presente estudo mostrou que a melhor maneira de avaliar corretamente o posicionamento do componente acetabular após um ATQ é medindo a anteversão e a abdução em uma radiografia AP após confirmar, em uma radiografia crosstable, que o componente não é retrovertido.

\section{Suporte Financeiro}

Não houve suporte financeiro de fontes públicas, comerciais, ou sem fins lucrativos.

Conflito de Interesses

Os autores não têm conflito de interesses a declarar.

\section{Referências}

1 Charnley J. Total hip replacement by low-friction arthroplasty. Clin Orthop Relat Res 1970;72(72):7-21

2 Del Schutte H Jr, Lipman AJ, Bannar SM, Livermore JT, Ilstrup D, Morrey BF. Effects of acetabular abduction on cup wear rates in total hip arthroplasty. J Arthroplasty 1998;13(06):621-626

3 Widmer KH, Zurfluh B. Compliant positioning of total hip components for optimal range of motion.J Orthop Res 2004;22(04):815-821

4 Woo RY, Morrey BF. Dislocations after total hip arthroplasty. J Bone Joint Surg Am 1982;64(09):1295-1306

5 Murray DW. The definition and measurement of acetabular orientation. J Bone Joint Surg Br 1993;75(02):228-232

6 Biedermann R, Tonin A, Krismer M, Rachbauer F, Eibl G, Stöckl B. Reducing the risk of dislocation after total hip arthroplasty: the effect of orientation of the acetabular component. J Bone Joint Surg Br 2005;87(06):762-769

7 Lewinnek GE, Lewis JL, Tarr R, Compere CL, Zimmerman JR. Dislocations after total hip-replacement arthroplasties. J Bone Joint Surg Am 1978;60(02):217-220

8 Ackland MK, Bourne WB, Uhthoff HK. Anteversion of the acetabular cup. Measurement of angle after total hip replacement. J Bone Joint Surg Br 1986;68(03):409-413

9 Müller O, Reize P, Trappmann D, Wülker N. Measuring anatomical acetabular cup orientation with a new X-ray technique. Comput Aided Surg 2006;11(02):69-75

10 Nho JH, Lee YK, Kim HJ, Ha YC, Suh YS, Koo KH. Reliability and validity of measuring version of the acetabular component. J Bone Joint Surg Br 2012;94(01):32-36

11 Visser JD, Konings JG. A new method for measuring angles after total hip arthroplasty. A study of the acetabular cup and femoral component. J Bone Joint Surg Br 1981;63B(04):556-559

12 Widmer $\mathrm{KH}$. A simplified method to determine acetabular cup anteversion from plain radiographs. J Arthroplasty 2004;19(03): 387-390

13 Arai N, Nakamura S, Matsushita T. Difference between 2 measurement methods of version angles of the acetabular component. J Arthroplasty 2007;22(05):715-720

14 Coventry MB. Late dislocations in patients with Charnley total hip arthroplasty. J Bone Joint Surg Am 1985;67(06):832-841

15 Seki M, Yuasa N, Ohkuni K. Analysis of optimal range of socket orientations in total hip arthroplasty with use of computer-aided design simulation. J Orthop Res 1998;16(04):513-517

16 Abdel MP, von Roth P, Jennings MT, Hanssen AD, Pagnano MW What Safe Zone? The Vast Majority of Dislocated THAs Are Within the Lewinnek Safe Zone for Acetabular Component Position. Clin Orthop Relat Res 2016;474(02):386-391

17 Esposito CI, Gladnick BP, Lee YY, et al. Cup position alone does not predict risk of dislocation after hip arthroplasty. J Arthroplasty 2015;30(01):109-113

18 Jolles BM, Zangger P, Leyvraz PF. Factors predisposing to dislocation after primary total hip arthroplasty: a multivariate analysis. J Arthroplasty 2002;17(03):282-288

19 Ha YC, Yoo JJ, Lee YK, Kim JY, Koo KH. Acetabular component positioning using anatomic landmarks of the acetabulum. Clin Orthop Relat Res 2012;470(12):3515-3523

20 Bhaskar D, Rajpura A, Board T. Current Concepts in Acetabular Positioning in Total Hip Arthroplasty. Indian J Orthop 2017;51 (04):386-396

21 McArthur B, Cross M, Geatrakas C, Mayman D, Ghelman B. Measuring acetabular component version after THA: CT or plain radiograph? Clin Orthop Relat Res 2012;470(10):2810-2818 